\title{
Application of RRLC-QTOF-MS-based metabonomics and UPE for investigating Spleen-Qi deficiency syndrome with Panax ginseng treatment
}

\author{
Ni Wang ${ }^{\mathrm{a}}$, Xin Huang ${ }^{\mathrm{a}, *}$, Tie $\mathrm{Li}^{\mathrm{b}}$, Mei Wang ${ }^{\mathrm{c}}$, Hao Yue ${ }^{\mathrm{a}}$, Changbao Chen ${ }^{\mathrm{a}}$, Shuying Liu ${ }^{\mathrm{a}, \mathrm{d}}$ \\ a Jilin Ginseng Academy, Changchun University of Chinese Medicine, Changchun, PR China \\ ${ }^{\mathrm{b}}$ Department of Acupuncture and Moxibustion, Changchun University of Chinese Medicine, Changchun, PR China \\ ${ }^{\mathrm{c}}$ LU-European Center for Chinese Medicine and Natural Compounds, Leiden University, Leiden, the Netherlands \\ ${ }^{\mathrm{d}}$ Changchun Institute of Applied Chemistry, Chinese Academy of Sciences, Changchun, PR China
}

\section{A R T I C L E I N F O}

\section{Keywords:}

Spleen-qi deficiency syndrome

Metabonomics

Ultraweak photon emission

Panax ginseng

\begin{abstract}
A B S T R A C T
Ethnopharmacological relevance: Spleen-Qi deficiency is a syndrome of traditional Chinese medicine. Panax ginseng (ginseng) is well known as the key herb for replenishing Qi and tonifying Spleen. However, the pathogenesis of Spleen-Qi deficiency syndrome and therapeutic mechanism of ginseng on Spleen-Qi deficiency constitution have not yet been entirely elucidated.

Aim of the study: The aim of this work was to investigate the pathogenesis of Spleen-Qi deficiency syndrome and therapeutic mechanism of ginseng on Spleen-Qi deficiency constitution.

Materials and methods: The urinary metabonomics was investigated before and after ginseng treatment in Spleen-Qi deficiency subjects by rapid resolution liquid chromatography coupled with quadrupole-time-of-flight mass spectrometry (RRLC-QTOF-MS). The spectra of metabolites were analyzed by principal component analysis (PCA) and the score showed significant difference among the three different groups. The serum biochemical parameters creatine kinases (CK), lactate dehydrogenase (LDH), interferon- $\gamma$ (INF- $\gamma$ ), Interleukin-4 (IL-4), superoxide dismutase (SOD) and malondialdehyde (MDA) were determined by assay kits. In addition, ultraweak photon emission (UPE) intensity was measured at dazhui point in each subject.

Results: According to the orthogonal partial least-squares discriminant analysis (OPLS-DA) results and biochemistry databases searching, 15 potential biomarkers were identified to be involved in Spleen-Qi deficiency syndrome and ginseng influenced Spleen-Qi deficiency. The metabonomics and biological experiment data indicated that metabolism pathways were corresponding to energy metabolism, amino acid, carbohydrate, lipid and pyrimidine metabolisms, oxidative stress urea cycle, and intestinal flora metabolism. And the UPE intensity decreased significantly after the treatment.

Conclusions: Ginseng could promote the related metabolisms in Spleen-Qi deficiency constitution and the metabolites and UPE intensity may be useful as potential biomarkers for diagnosing and monitoring for Spleen-Qi deficiency syndrome.
\end{abstract}

\section{Introduction}

Spleen-Qi deficiency is a syndrome of traditional Chinese medicine, is characterized by weak breath, body and viscera hypo function (Wu et al., 2017). The clinical manifestations of Qi deficiency include physical weakness, pale, shortness of breath, sweating, low voice etc. It has a close relationship with congenitally deficient, fatigue and malnutrition, etc. (Wang et al., 2018). This sub healthy constitution could be regulated in traditional Chinese medicine clinic and will be one of the emphases on the prevention for treatment of disease (Li et al., 2012). Panax ginseng (ginseng) is well known as the key herb for replenishing Qi and tonifying Spleen. It has been applied in treatment of any deficiency of vital energy, blood and body fluid and to enhance physical function (Kang et al., 2012; Lin et al., 2016; Mancuso et al.,

Abbreviations: RRLC-QTOF-MS, rapid resolution liquid chromatography coupled with quadrupole-time-of-flight mass spectrometry; PCA, principal component analysis; CK, creatine kinases; LDH, lactate dehydrogenase; INF- $\gamma$, interferon- $\gamma$; IL-4, Interleukin-4; SOD, superoxide dismutase; MDA, malondialdehyde; UPE, ultraweak photon emission; OPLS-DA, orthogonal partial least-squares discriminant analysis; ROS, reactive oxygen species; QC, quality control; BPI, base peak intensity; VIP, variable importance in the projection; FAD, flavin adenine dinucleotide

${ }^{*}$ Corresponding author. Jilin Ginseng Academy, Changchun University of Chinese Medicine, Changchun, 130117, PR China.

E-mail address: huangxin@ccucm.edu.cn (X. Huang). 
2017; Zhao et al., 2015). However, the therapeutic mechanism of ginseng on Spleen Qi deficiency constitution has not yet been entirely elucidated.

Metabonomics study the physical and functional changes of the body through monitoring the endogenous metabolites (Cao et al., 2018; Cui et al., 2017; Liu et al., 2018). Metabonomics regards human body as a whole, and the physiological and pathological process are dynamic. This concept is consistent with "wholeness", "dynamic concept" and "dialectics" of the traditional Chinese medicine theory (Liu et al., 2012; Xu et al., 2018). Ultraweak Photon Emission (UPE) occurs spontaneously in living organisms, without the need for external intervention. The source of UPE is closely related to the electronic transport and the generation of reactive oxygen species (ROS) during oxidative metabolic processes. Biological ROS are produced dynamically during chemical metabolic redox reactions, including lipid peroxidation and protein/ nucleic acid generation. Moreover, during these metabolic processes, electrons can become excited, and energy is emitted in the form of photons (Ives et al., 2014; Liu, 2013). Therefore, UPE can be measured in order to detect the physiological state of the human body and to measure dynamic changes in health (Guo et al., 2018). The link between human photon emission and disease has been studied revealing several interesting observations based on the intensity of the signal (Cifra et al., 2014; Sun et al., 2017).

In this study, RRLC-QTOF-MS was applied to obtain global endogenous metabolites in urine of Spleen-Qi deficiency subjects and ginseng effects on metabolites changes. The serum biochemical parameters CK, LDH, INF- $\gamma$, IL-4, SOD and MDA exhibited the regulation of ginseng on Spleen-Qi deficiency. Meanwhile, we employed a UPE strategy on dazhui point in each subject to evaluate the intensity responses of ginseng treatment. Combined the pattern recognition method, the metabolic pathways and mechanism of Spleen-Qi deficiency syndrome and ginseng treating Spleen-Qi deficiency were summerized. The possibility of integrated UPE and metabonomics for a personalized monitoring of changes of the system towards health or disease were discussed.

\section{Materials and methods}

\subsection{Chemicals, reagents and materials}

Acetonitrile (HPLC grade, Fisher Scientific), formic acid (HPLC grade, Sigma-Aldrich), ultrapure water $(18 \mathrm{M} \Omega / \mathrm{cm}$, Milli-Q water system). Superoxide dismutase (SOD), malondialdehyde (MDA), creatine kinases (CK), lactate dehydrogenase (LDH), interferon- $\gamma$ (INF- $\gamma$ ) and Interleukin-4 (IL-4) assay kits (Nanjing Jiancheng Bioengineering Institute).

Panax ginseng C.A. Mey. was purchased from Fusong (Jilin, China) and authenticated by Professor Shumin Wang (Changchun University of Chinese Medicine, Changchun, China). The quality of ginseng crude roots was controlled and processed according to the Chinese Pharmacopoeia (2015). The voucher specimens were stored in Changchun University of Chinese Medicine, Changchun, China (No. 20180506). Ginseng capsule (Batch No. 180611) and placebo capsule (Batch No. 180602) were provided by Jilin Zixin Pharmaceutical Industrial Co., Ltd. A ginseng capsule contained ginseng powder $375 \mathrm{mg}$ and a placebo capsule contained corn starch with identically color, shape, and taste.

\subsection{Study design and subjects}

This study was conducted in accordance with the Declaration of Helsinki and Tokyo, and with ethical approval from the Ethics Committee at the First Affiliated Hospital of Changchun University of Chinese Medicine (CCZYFYLL2018-084-2). The subjects were recruited from affiliated hospital of Changchun university of Chinese medicine through advertisements. All participants provided written informed consent and were screened by physical examination, anamnesis evaluation and clinical laboratory test. The subjects were declared eligible, while meeting the inclusion and exclusion criteria and the diagnosis of Chinese medicine physicians. On that basis, fifteen general subjects were designed as health group and thirty Spleen-Qi deficiency subjects were randomly and equally assigned into placebo and ginseng groups. The ginseng group took 4 ginseng capsules twice per day for 4 weeks, and the placebo group was provided with 4 placebo capsules twice per day for 4 weeks. The first void urine samples of subjects were collected into tubes after an overnight fast at the Zeroth and Fourth weeks, respectively and stored frozen at $-80{ }^{\circ} \mathrm{C}$. In addition, UPE measurements were also carried out at the same time.

\subsection{RRLC-QTOF-MS condition and urine analysis}

1200 series RRLC (Agilent Technologies, Santa Clara California, USA) consists of a quaternary gradient pump, an autosampler, and a thermostatically column compartment, coupled to Agilent 6520 Q-TOF Mass spectrometer (Agilent Technologies, Santa Clara California, USA) and controlled with MassHunter system software. Reversed-phase column (Eclipse Plus C18, $2.1 \times 150 \mathrm{~mm}, 3.5 \mu \mathrm{m}$; Agilent Technologies, Santa Clara California, USA) was used for separations.

The mass spectrometry equipped with electrospray ionization (ESI) was operated under positive and negative ion mode. The data were acquired in centroid scan mode with normal scan rate and $m / z$ 50.0-1000.0 scan range. According to standard calibration procedure, the mass scale was calibrated prior to detection. Key operating parameters were: gas temperature $350{ }^{\circ} \mathrm{C}$, drying gas $9 \mathrm{~L} / \mathrm{min}$, nebulizer 40 psig, fragmentor $150 \mathrm{~V}$, skimmer $65 \mathrm{~V}$, and capillary $+3500 \mathrm{~V}$.

The mobile phases were $0.1 \%$ formic acid in acetonitrile (solvent A) and $0.1 \%$ formic acid in ultrapure water (solvent $\mathrm{B}$ ). The gradient elution program with $0.2 \mathrm{~mL} / \mathrm{min}$ flow rate as follows: $0-5 \min 5-20 \%$ A, 5-10 min 20-35\% A, 10-15 min 35-100\% A, 15-17 min held at $100 \%$ A. The temperature of autosampler and column were set at $15{ }^{\circ} \mathrm{C}$ and $30{ }^{\circ} \mathrm{C}$, respectively. The flow rate was set at $0.3 \mathrm{~mL} / \mathrm{min}$ and the injection volume was $5 \mu \mathrm{L}$.

The system consistency and method validation were conducted by quality control (QC) sample, run before the sample sequence and every ten samples during analysis.

Prior to RRLC-QTOF-MS analysis, urine samples were thawed at room temperature, centrifuged at $3000 \times g$ for $10 \mathrm{~min}$ at $4{ }^{\circ} \mathrm{C}$ and filtered through a $0.22 \mu \mathrm{m}$ membrane filter.

\subsection{Serum biochemical parameters analysis}

The whole blood of subjects was centrifuged for $10 \mathrm{~min}$ at $3000 \times \mathrm{g}$ and $4{ }^{\circ} \mathrm{C}$ to get the serum. The serum samples were stored at $-80{ }^{\circ} \mathrm{C}$ and thawed at $4{ }^{\circ} \mathrm{C}$, prior to analysis. $\mathrm{CK}, \mathrm{LDH}$ and SOD activities and MDA, INF- $\gamma$ and IL- 4 levels were measured using commercially and ELISA kits.

\subsection{UPE condition and analysis}

UPE system (Meluna, Geldermalsen, the Netherlands) includes a 9235QA photomultiplier tube (ET Enterprises, Uxbridge, UK). The PMT fitted with a $50 \mathrm{~mm}$ diameter window, with spectral in the range of $150-700 \mathrm{~nm}$ and the background noise was also measured in 5-6 counts/s. The subjects should lie down well in a dark chamber, maintained temperature at $20 \pm 1.0{ }^{\circ} \mathrm{C}$. And the same areas on Dazhui point were measured with PMT operated in the single-photon counting mode. The distance between the PMT and skin was $30 \mathrm{~mm}$. UPE signals were recorded between 9 a.m. and 3 p.m. in order to minimize any possible effects of diurnal rhythms using a model 6602 PCI card (National Instruments, Austin, TX). Each signal was recorded for $20 \mathrm{~min}$ by counting the number of photons emitted in 6000 consecutive $50 \mathrm{~ms}$ bins. 


\subsection{Data analysis}

The RRLC-QTOF-MS raw data were processed with Mass Profiler Professional statistic software (Agilent Technologies, Santa Clara California, USA) for peak identification and normalization. To compare the metabolite profiles of the subjects' groups, normalized peak areas were analyzed by principal component analysis (PCA) and orthogonal partial least square discriminant analysis (OPLS-DA) using SIMCA-P Statistics 13.0 software. A $t$-test between groups of independent samples was used to evaluate the significant difference $(P<0.05)$ using SPSS Statistics 13.0 software. For identification of potential markers, the following databases were used: Massbank (http://www.massbank.jp), HMDB (http://www.hmdb.ca/), and KEGG (http://www.kegg.com/).

\section{Results and discussion}

\subsection{Metabolites profiles of RRLC-QTOF-MS}

The validation of RRLC-QTOF-MS method was conducted using QC sample of the urine metabolites in the positive and negative ion mode. The base peak intensity (BPI) chromatograms of the same sample in both ion modes were shown in Fig. 1.

The system consistency was evaluated by the RSDs \% of retention times $0.3-1.0 \%$ and peak areas $1.2-2.4 \%$ of selected ions. These results demonstrated that the RRLC-QTOF-MS method developed with good stability and reproducibility could be applied for metabonomics analysis.

The RRLC-QTOF-MS data were processed by Mass Hunter Qualitative Analysis and Mass Profiler Professional statistic software. And then the processed data were investigated using pattern recognition method OPLS-DA. In the OPLS-DA score plots (Fig. 2), the three groups separated clearly in both positive and negative ion modes. This result indicated that there was little difference within the group and the urinary metabolic pattern changed significantly with the ginseng treatment in Spleen-Qi deficiency subjects. The ginseng treatment group was close to health group, demonstrated that the metabolic profile of ginseng treatment group was changed to health group.

\subsection{Potential biomarker identification}

After OPLS-DA processing, metabolites contributing to the clustering and discrimination were generated according to a threshold of variable importance in the projection (VIP) values. As the VIP value above 1.2 and $p$-value of $t$-test below 0.05 , the ions were considered as potential biomarkers. And the identifications of potential biomarkers were based on their accurate quasi-molecular ion and MS/MS fragmentation patterns compared with available reference standards and/ or free database resources. Meanwhile, by the discrimination of health group, ginseng treatment group and Spleen-Qi deficiency group using the OPLS-DA model, totally 15 endogenous metabolites were shown as urinary potential biomarkers correlated with the treatment of ginseng in Spleen-Qi deficiency. The identified potential biomarkers were summarized in Table 1.

To illustrate the identification process of biomarkers, took $\mathrm{m} / \mathrm{z}$ 183.0455 ion at $2.55 \mathrm{~min}$ in negative ion mode as an example. The $\mathrm{MS}^{2}$ spectrum of $m / z 183.0455$ ion was shown in Fig. 3. The possible elemental compositions $\mathrm{C}_{6} \mathrm{H}_{6} \mathrm{~N}_{2} \mathrm{O}_{2}$ of this compound were calculated by the measured $m / z$ value and compared with reference compounds registered in the database. And then the fragment ions $m / z 137.9441$ and $\mathrm{m} / z 119.2425$ and their corresponding intensities were compared with those of reference compounds registered in the database. Based on the above process information, the biomarker was identified as urocanic acid. The other compounds were identified as this process method depending on the elemental composition in searching databases. The trends of the identified biomarkers changed during ginseng treatment in Spleen-Qi deficiency were shown in Table 1.

\subsection{Mechanism interpretation of ginseng on Spleen-Qi deficiency}

In this study, 15 potential biomarkers were identified to explain the effect of ginseng on Spleen-Qi deficiency and the concentration changes of these metabolites between treatment and non-treatment groups were described in Table 1. And the correlation network of urinary biomarkers was shown in Fig. 4.

Through the kynurenic acid pathway, tryptophan mainly produces acetyl-CoA, enters the tricarboxylic acid cycle for complete oxidation and energy supply. And some tryptophan was produced to indole by intestinal bacteria (Badawy, 2017). In the Spleen-Qi deficiency group, the concentrations of tryptophan and indole derivatives, indoleacetyl glutamine, were increased. The content of xanthurenic acid, the derivative in kynurenic acid metabolic pathway, was decreased. The concentration changes of these amino acid indicated the accumulation of tryptophan, kynurenic acid metabolism weakened and indole metabolism strengthened. Therefore, the energy metabolism was disturbed and metabolism of intestinal bacteria was disordered.

Glycocholic acid is one of the binding cholic acids formed by the combination of cholic acid and glycine. In healthy condition, bile acids secret by bile entered the intestinal tract and most of them are mixed with lipid products to form microclusters and thus promotes lipid absorption. Only a fraction of bile acids is excreted to the outside of body (Shen et al., 2019; Taylor et al., 2018). Increased glycocholic acid in Spleen-Qi deficiency group also demonstrated the abnormal intestinal digestion.

Epinephrine is a peripheral neurotransmitter, of catecholamine and
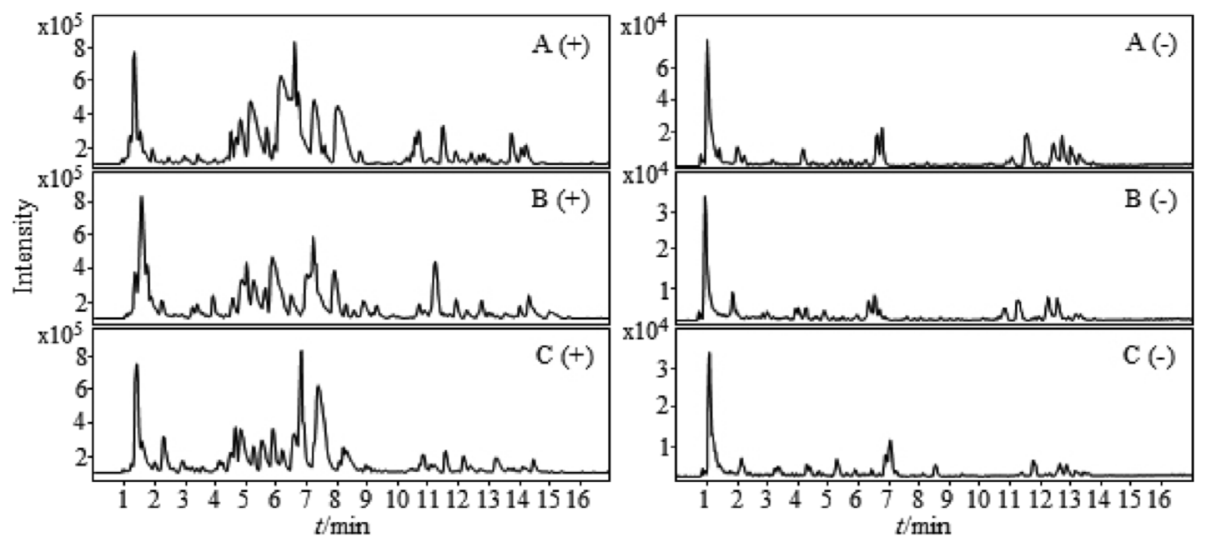

Fig. 1. Base peak intensity (BPI) chromatogram in positive and negative ion modes of urine sample from health group (A), ginseng treatment group (B) and Spleen-Qi deficiency group (C). 

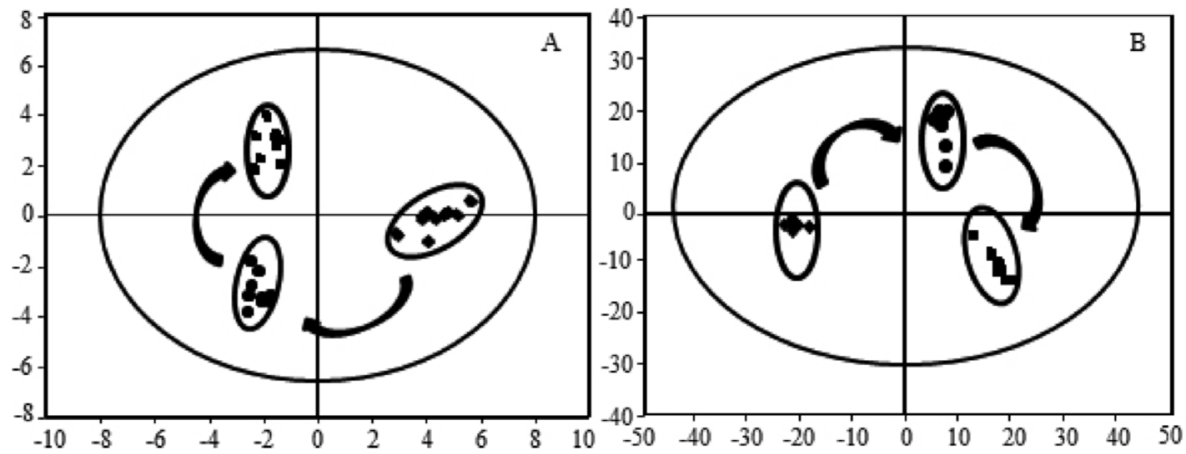

Fig. 2. Results of OPLS-DA analysis, (A) scores plot in positive mode; (B) scores plot in negative mode of Spleen-Qi deficiency group ( $\bullet$ ), ginseng treatment group ( and health group (

Table 1

Potential biomarkers of Spleen-Qi deficiency syndrome.

\begin{tabular}{|c|c|c|c|c|}
\hline $\begin{array}{l}\text { Retention } \\
\text { time/min }\end{array}$ & $m / z$ & Metabolite & Formula & $\begin{array}{l}\text { Change } \\
\text { trends }\end{array}$ \\
\hline \multicolumn{5}{|l|}{$+\mathrm{ESI}$} \\
\hline 1.57 & 164.0412 & Acetylcysteine & $\mathrm{C}_{5} \mathrm{H}_{9} \mathrm{NO}_{3} \mathrm{~S}$ & Up \\
\hline 4.51 & 184.1132 & Epinephrine & $\mathrm{C}_{9} \mathrm{H}_{13} \mathrm{NO}_{3}$ & Down \\
\hline 7.06 & 179.0477 & Gluconolactone & $\mathrm{C}_{6} \mathrm{H}_{10} \mathrm{O}_{6}$ & Down \\
\hline \multicolumn{5}{|l|}{-ESI } \\
\hline 1.77 & 130.0249 & 2-Oxosuccinamate & $\mathrm{C}_{4} \mathrm{H}_{5} \mathrm{NO}_{4}$ & Down \\
\hline 1.96 & 264.0273 & Xanthurenic acid & $\mathrm{C}_{10} \mathrm{H}_{7} \mathrm{NO}_{4}$ & Down \\
\hline 2.55 & 183.0455 & Urocanic acid & $\mathrm{C}_{6} \mathrm{H}_{6} \mathrm{~N}_{2} \mathrm{O}_{2}$ & Down \\
\hline 5.78 & 503.1369 & $\begin{array}{l}\text { Reduced flavin } \\
\text { mononucleotide } \\
\text { (FMNH2) }\end{array}$ & $\mathrm{C}_{17} \mathrm{H}_{23} \mathrm{~N}_{4} \mathrm{O}_{9} \mathrm{P}$ & Up \\
\hline 6.67 & 447.9833 & Cytidine diphosphate & $\mathrm{C}_{9} \mathrm{H}_{15} \mathrm{~N}_{3} \mathrm{O}_{11} \mathrm{P}$ & Up \\
\hline 9.31 & 249.0917 & Tryptophan & $\mathrm{C}_{11} \mathrm{H}_{12} \mathrm{~N}_{2} \mathrm{O}_{2}$ & Up \\
\hline 9.38 & 218.1062 & Pantothenic acid & $\mathrm{C}_{9} \mathrm{H}_{17} \mathrm{NO}_{5}$ & Down \\
\hline 9.63 & 362.1416 & Indoleacetyl glutamine & $\mathrm{C}_{15} \mathrm{H}_{17} \mathrm{~N}_{3} \mathrm{O}_{4}$ & Up \\
\hline 9.95 & 386.1626 & $\begin{array}{l}\text { 6-(alpha-D- } \\
\text { Glucosaminyl)-1D-myo- } \\
\text { inositol }\end{array}$ & $\mathrm{C}_{12} \mathrm{H}_{23} \mathrm{NO}_{10}$ & Up \\
\hline 10.59 & 188.0987 & Homocitrulline & $\mathrm{C}_{7} \mathrm{H}_{15} \mathrm{~N}_{3} \mathrm{O}_{3}$ & Up \\
\hline 12.82 & 201.1272 & 2-Ethylsuberic acid & $\mathrm{C}_{10} \mathrm{H}_{18} \mathrm{O}_{4}$ & Up \\
\hline 15.64 & 524.3394 & Glycocholic acid & $\mathrm{C}_{26} \mathrm{H}_{43} \mathrm{NO}_{6}$ & Up \\
\hline
\end{tabular}

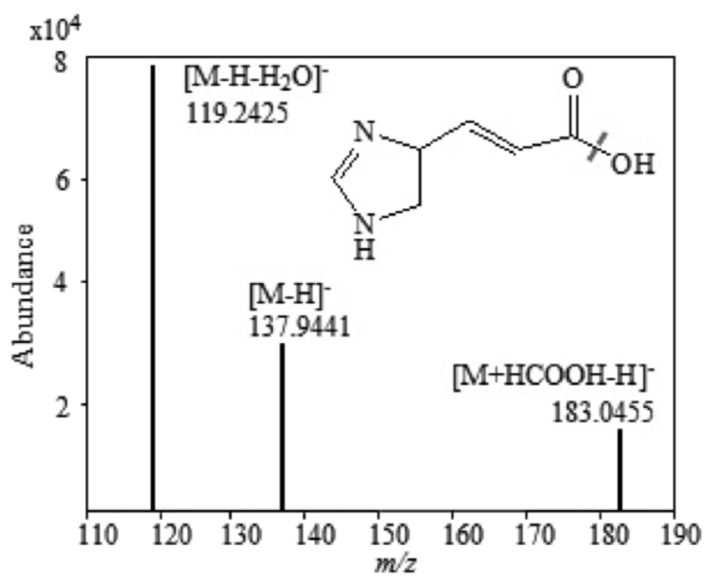

Fig. 3. The $\mathrm{MS}^{2}$ spectrum in negative mode of urocanic acid.

transformed from tyrosine. Epinephrine could increase glycogen hydrolysis to glucose to raise blood sugar levels and meanwhile promote lipids broken down in adipose cell to provide energy for the body. In Spleen-Qi deficiency group, epinephrine decreased lead to relatively hyperfunction of parasympathetic nerve and disturbance of blood glucose and energy metabolism. Thereby, Spleen-Qi deficiency presents the symptom of inappetence, fatigue, etc (Zhen et al., 2017).
Urocanic acid is the intermediate product in the conversion of histidine to glutamic acid (Cao et al., 2019). And glutamic acid interconverts with $\alpha$-ketoglutaric acid and enters the tricarboxylic acid cycle to provide energy. Glutamic acid with ammonia synthesizes glutamine and participates in ammonia transport. A decrease in urocanic acid in Spleen-Qi deficiency group causes the breakdown in glutamate production, tricarboxylic acid cycle and ammonia transport.

Another two biomarkers involve metabolic disorder of tricarboxylic acid cycle are 2-Oxosuccinate and FMNH2. 2-Oxosuccinate could converse to ketosuccinic acid, the intermediate in tricarboxylic acid cycle. In Spleen-Qi deficiency group, the concentration of 2-Oxosuccinate decreased to disturb tricarboxylic acid cycle. FMNH2 is the metabolic product of flavin adenine dinucleotide (FAD). In the tricarboxylic acid cycle, the dehydrogenation of succinic acid generates to fumaric acid and removed $2 \mathrm{H}$ shall be given to $\mathrm{FAD}$ to generate $\mathrm{FADH}_{2}$. Increased $\mathrm{FMNH}_{2}$ indicated that FAD metabolic disorder and then affected tricarboxylic acid cycle.

Homocitrulline, a metabonomic product of urea circulation disorder (Diego et al., 2015), could affect internal ammonia transport. The urea cycle is the main way of body to release ammonia. For the Spleen-Qi deficiency volunteers, increased homocitrulline demonstrated ammonia excretion disorder in this group.

Acetylcysteine is the N-acetyl derivative of L-cysteine and the precursor of glutathione (Birnie-Gauvin et al., 2017; Paschalis et al., 2018). Glutathione is an antioxidant, could eliminate free radical and improve the immunity of human (Niu et al., 2019). The concentration of acetylcysteine increased in the urine of Spleen-Qi deficiency subjects, indicated the precursor loss of glutathione. Therefore, the synthesis of glutathione was insufficient, caused the organism immunity reduced and the skin is dull.

In this study, the above biomarkers were improved in the ginseng treated Spleen-Qi deficiency group, suggested the important regulation for ginseng in amino acid metabolism, intestinal digestion and energy metabolism of Spleen-Qi deficiency constitution.

Gluconolactone is a lactone or oxidation derivative of glucose. The glucose can provide energy for the body through a variety of metabolism pathways, is the main source of energy for body. In the urine of Spleen-Qi deficiency group, the content of gluconolactone decreased, indicated the sugar metabolic disorder caused the lack of energy supply and infirmity of these volunteers.

2-Ethylsuberic acid is the ethyl ester of linoleic acid. 6-( $\alpha$-D-glucosaminyl)-1D-myoinositol is an intermediate in the formation of glycerol into triacylglycerol. Increased 2-ethylsuberic acid and 6-( $\alpha$-D-glucosaminyl)-1D-myoinositol in the urine of Spleen-Qi deficiency subjects, demonstrated metabolic abnormalities of fatty acid and lipid.

Pantothenic acid participates in the synthesis of HSCo A, plays an important role in the metabolism of sugar, fat and protein (Antonio et al., 2015). Decreased pantothenic acid in Spleen-Qi deficiency group indicated the energy metabolic disturbance.

Pyrimidine nucleotide is synthesized by pyrimidine ring and ribo-5- 


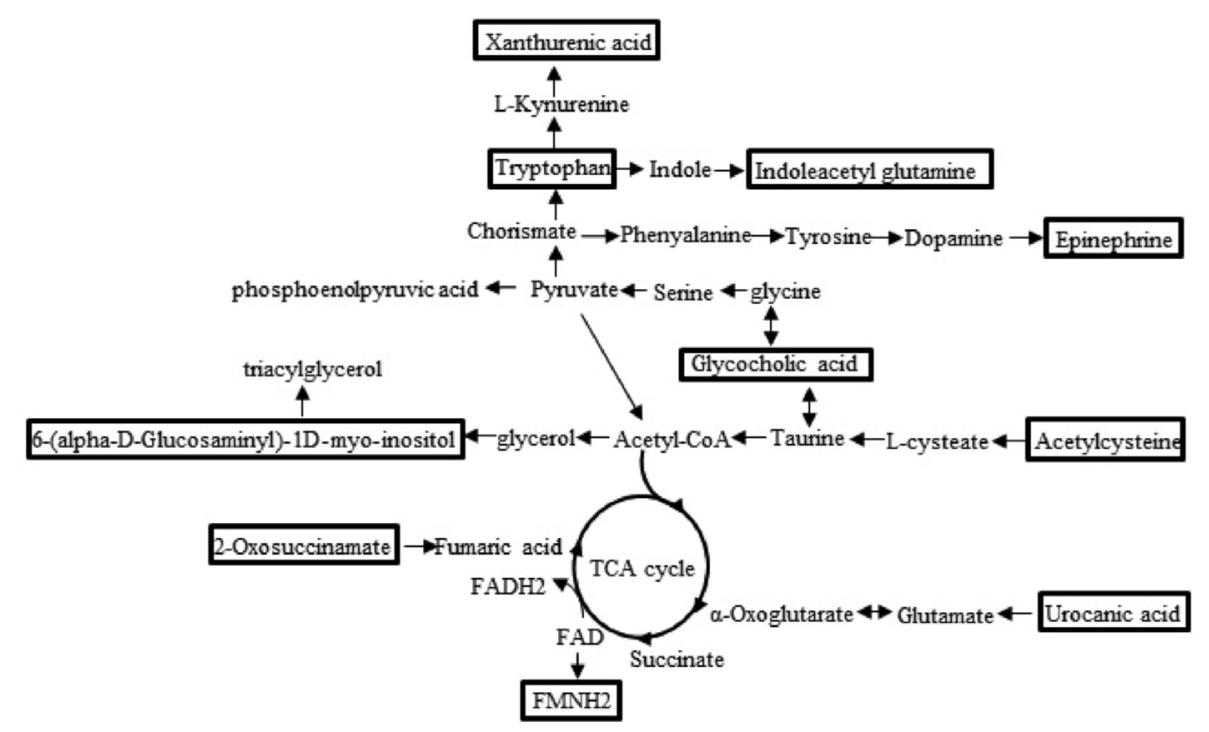

Fig. 4. Metabolic disturbance map of Spleen-Qi deficiency syndrome with identification markers in the square frame.

phosphate and transforms to cytoside triphosphate gradually. Cytidine diphosphate is the intermediate from pyrimidine nucleotide to cytoside triphosphate, increased in the urine of Spleen-Qi deficiency subjects and indicated the metabolic disorder of pyrimidine.

From the above results, after ginseng treatment, the biomarkers were improved for Spleen-Qi deficiency. It demonstrated that ginseng could regulate the metabolism of sugar, lipid, energy and pyrimidine of Spleen-Qi deficiency constitution.

\subsection{Indication of blood biochemical parameters}

SOD is the natural free radical scavenger in human body and catalyze superoxide anion free radical (Zhu, 2011). MDA is the final product of oxidative stress and its content can indirectly reflect the severity of free radical attack on body cells (Borut et al., 2013). The determination of SOD activity and MDA level can coordinate with each other to reflect the REDOX metabolism of the body. According to the results in Table 2, the content of MDA was significantly higher in Spleen-Qi deficiency group and the activity of SOD was lower than that in health group. It indicated that there was REDOX disorder in Spleen-Qi deficiency constitution.

After ginseng treating, MDA level decreased and SOD activity increased remarkably. It showed that ginseng could increase the antioxidant activity and inhibit the lipid peroxidation reaction in Spleen-Qi deficiency constitution and so presented regulation on the REDOX balance of the body. Combined with the above metabolomics analysis, Spleen-Qi deficiency constitution may have glutathione metabolism disorder and leading to antioxidant dysfunction. And ginseng could interfere the abnormal metabolism of antioxidation caused by glutathione in the body.

CK is commonly found in animal skeletal muscle, heart and brain tissue, is an important kinase involved in cell energy transport, muscle contraction and ATP regeneration (Xiong, 2012). The change of CK activity can reflect the energy metabolism in the body. LDH is an important enzyme system in glycolysis pathway and the quality and quantity change of it directly affects energy metabolism of organism. In this research, the activity of CK in serum of Spleen-Qi deficiency group was obviously higher than that in health group and the activity of LDH decreased significantly (Table 2). It revealed that Spleen-Qi deficiency constitution have abnormal energy metabolism compared with healthy people. After ginseng treatment, the activities of CK and LDH in serum of Spleen-Qi deficiency group presented significant decreased and increased, respectively. It showed that ginseng could regulate energy metabolism of Spleen-Qi deficiency constitution. According to the metabolomics results of this research, ginseng can regulate the metabolism of pantothenic acid, riboflavin, dihydroxyacetone phosphate and carnitine, so as to improve the sugar, fatty acid and energy metabolism of Spleen-Qi deficiency constitution.

IFN- $\gamma$ and IL-4 are intracellular factors with immunomodulatory effects and both of them play an important role in immune response (Xing et al., 2015; Yang et al., 2010). The ELISA reagent kits results showed that the level of IFN- $\gamma$ decreased obviously in serum of SpleenQi deficiency constitution. And for IL-4, the concentration in serum was significantly higher than that in healthy group. After treatment with ginseng, the content of IFN- $\gamma$ increased and that of IL-4 reduced significantly. It indicates that Spleen-Qi deficiency constitution present immune system disorder and the ginseng can regulate the immune system. From the results of metabolomics, ginseng may play the therapeutic role in Spleen-Qi deficiency by interfering in the metabolism of glutathione arachidonic acid and canine urine.

\subsection{UPE intensity analysis}

The UPE intensities were measured on Dazhui point of subjects in the single photon counting mode and were accumulated for $20 \mathrm{~min}$. The UPE intensities changes among the three groups before and after

Table 2

The biochemical parameters of serum sample of Spleen-Qi deficiency syndrome before and after treatment of ginseng.

\begin{tabular}{|c|c|c|c|c|c|c|}
\hline Group & $\mathrm{CK}(\mathrm{U} / \mathrm{mL})$ & LDH (U/L) & $\mathrm{IL}-4(\mathrm{pg} / \mathrm{mL})$ & INF- $\gamma(\mathrm{pg} / \mathrm{mL})$ & $\mathrm{SOD}(\mathrm{U} / \mathrm{mL})$ & MDA (nmol/mL) \\
\hline HG & $0.076 \pm 0.02$ & $2639.96 \pm 169.84$ & $3.64 \pm 0.25$ & $178.3 \pm 14.03$ & $168.69 \pm 8.31$ & $2.32 \pm 0.22$ \\
\hline SQDG & $1.04 \pm 0.16^{* *}$ & $1790.90 \pm 73.45^{* *}$ & $6.29 \pm 0.48^{* *}$ & $71.71 \pm 9.65^{* *}$ & $124.83 \pm 1.64 * *$ & $3.79 \pm 0.32^{* *}$ \\
\hline GTG & $0.17 \pm 0.03^{* *}$ & $2144.51 \pm 53.11^{* *}$ & $4.34 \pm 0.23^{*}$ & $147.48 \pm 8.77^{*}$ & $134.89 \pm 2.37^{* *}$ & $2.72 \pm 0.16^{*}$ \\
\hline
\end{tabular}

HG: health group; SQDG: Spleen-Qi deficiency group; GTG: ginseng treatment group.

$\left(^{* *}: p<0.001 ; *: p<0.01\right)$. 


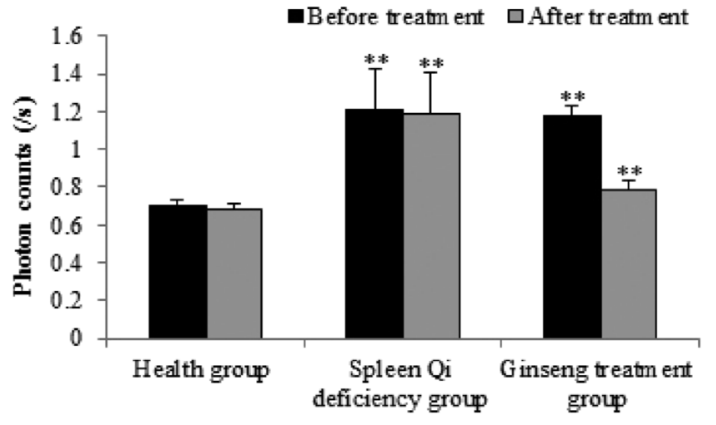

Fig. 5. Comparison of UPE data acquisition intensity before and after treatment in health group, Spleen-Qi deficiency group and ginseng treatment group. $* *$ : $p<0.001$ vs Spleen-Qi deficiency group.

ginseng treatment were presented in Fig. 5.

The UPE intensities of Spleen-Qi deficiency group were significantly higher than the health group. And for Spleen-Qi deficiency group, the UPE intensities significantly decreased after ginseng treatment. It demonstrated that the body's ability to store photons decreases under the condition of Spleen-Qi deficiency. According to the results of metabonomics analysis, the glutathione, amino acid, energy and lipid metabolisms were disturbed under Spleen-Qi deficiency, which led to abnormal metabolic reactions such as REDOX in vivo and the intensity of UPE increased significantly. While after ginseng treatment, the oxidative stress was inhibited and the UPE intensity was decreased.

\section{Conclusion}

The Spleen-Qi deficiency syndrome and treatment of ginseng on Spleen-Qi deficiency was first investigated by urinary metabonomics and UPE at dazhui point of the subjects. Fifteen potential biomarkers were identified to be involved in Spleen-Qi deficiency syndrome and ginseng regulating Spleen-Qi deficiency, participating energy metabolism, amino acid, carbohydrate, lipid and pyrimidine metabolisms, urea cycle and intestinal flora metabolism. The ginseng presents replenishing Qi and tonifying spleen effect by influencing the above metabolism pathways. In the results of serum biochemical parameters detection, ginseng could regulate the antioxidant activities, energy metabolism and immune system disorder of Spleen-Qi deficiency constitution. According to UPE result, the intensity at dazhui point of Spleen-Qi deficiency subjects were higher than that of health ones. After ginseng treatment, the UPE intensity decreased significantly indicated that ginseng could promote the related metabolisms and reduce the oxidative stress of Spleen-Qi deficiency subjects. And the metabolites and UPE intensity may be useful as potential markers for diagnosing and monitoring for Spleen-Qi deficiency syndrome.

\section{CRediT authorship contribution statement}

Ni Wang: Data curation, Formal analysis. Xin Huang: Data curation, Formal analysis. Tie Li: Formal analysis. Mei Wang: Methodology. Hao Yue: Data curation, Formal analysis. Changbao Chen: Project administration. Shuying Liu: Writing - review \& editing.

\section{Declaration of competing interest}

The authors declare that they have no competing interests.

\section{Acknowledgements}

This work was supported by the National Key R\&D Program of the Ministry of Science and Technology (2017YFC1702105), Science and Technology Development Plan Project of Jilin Province
(20170414027GH), and the Cultivation Fund Project of Changchun University of Chinese Medicine (2018KJ17).

\section{Appendix A. Supplementary data}

Supplementary data to this article can be found online at https:// doi.org/10.1016/j.jep.2020.112822.

\section{References}

Antonio, S., Javier, R.G., Julian, C., Luis, A., 2015. Pantothenic acid: an overview focused on medical aspects. Eur. Sci. J. 11 (21), 1-18.

Badawy, A.B., 2017. Kynurenine pathway of tryptophan metabolism: regulatory and functional aspects. Int. J. Tryptophan Res. 10 (1), 1-20.

Birnie-Gauvin, K., Larsen, M.H., Aarestrup, K., Willmore, W.G., Cooke, S.J., 2017. Nacetylcysteine manipulation fails to elicit an increase in glutathione in a teleost model. Fish Physiol. Biochem. 44, 137-142.

Borut, P., Dušan, š., Irina, M., 2013. Achieving the balance between ROS and antioxidants: when to use the synthetic antioxidants. Oxid. Med. Cell. Longev. 1-11 2013.

Cao, J., Mi, Y.J., Shi, C.L., Bian, Y.C., Huang, C.R., Ye, Z.J., Liu, L.S., Miao, L.Y., 2018. First-line anti-tuberculosis drugs induce hepatotoxicity: a novel mechanism based on a urinary metabolomics platform. Biochem. Bioph. Res. Co. 497 (2), 485-491.

Cao, J., She, L., Song, Y., 2019. The glutamate biosynthetic pathway in brain: a novel mechanism of moderate UV-induced neurobehavioral changes. Acta Biochim. Biophys. Sin. 51 (2), 227-228.

Chinese Pharmacopoeia Commission, 2015. China Pharmacopoeia. pp. 81-82.

Cifra, M., Pospíšil, P., 2014. Ultra-weak photon emission from biological samples: definition, mechanisms, properties, detection and applications. J. Photochem. Photobiol. B Biol. 139, 2-10.

Cui, Y., Guo, H., Kuang, H.X., Wang, Q.H., 2017. Overview on application of metabonomics in study on traditional Chinese medicine syndromes. Chin. J. Tradit. Chin. Med. Pharm. 32 (2), 672-675.

Diego, M., Daria, D., Emanuela, P., Magnus, M., Sara, B., Enrico, B., Giuseppe, F., Carlo, D.V., 2015. The hyperornithinemia-hyperammonemia-homocitrullinuria syndrome. Orphanet J. Rare Dis. 10 (1), 1-17.

Guo, L., Guo, Y., Zhang, G.X., Zhao, X.W., Fan, Z.J., Zhi, M.J., Li, T., Wang, F.C., 2018. Biological Ultra-weak luminescence and its application to research of acupuncture. Acupunct. Res. 43 (6), 384-387.

Ives, J.A., van Wijk, E.P.A., Bat, N., Crawford, C., Walter, A., Jonas, W.B., van Wijk, R., van der Greef, J., 2014. Ultraweak photon emission as a non-Invasive health assessment: a systematic review. PloS One 9 (2), e87401.

Kang, S., Min, H., 2012. Ginseng, the 'immunity boost': the effects of Panax ginseng on immune system. J. Ginseng. Res. 36 (4), 354-368.

Li, Y.F., Yin, Y., Jin, P., 2012. Research progress of Qi deficiency constitution. Acta. Chin. Med. Pharm. 40 (1), 90-92.

Lin, H., Pi, Z.F., Men, L.H., Chen, W.J., Liu, Z.Q., Liu, Z.Y., 2016. Urinary metabonomic study of Panax ginseng in deficiency of vital energy rat using ultra performance liquid chromatography coupled with quadrupole time-of-flight mass spectrometry. J. Ethnopharmacol. 184, 10-17.

Liu, D.S., Zhao, N.N., Wang, M.G., Pi, X., Feng, Y., Wang, Y., Tong, H.S., Zhu, L., Wang, C.S., Li, E.Y., 2018. Urine volatile organic compounds as biomarkers for minimal change type nephrotic syndrome. Biochem. Bioph. Res. Co. 496 (1), 58-63.

Liu, G.F., 2013. Advances in Ultra-weak bioluminescence technology. Biol. Teaching. 38 (6), 2-3.

Liu, P., Liu, S., Tian, D., Wang, P.Y., 2012. The applications and obstacles of metabonomics in traditional Chinese medicine. Evid. Based. Compl. Alt. Med. 1-4 2012.

Mancuso, C., Santangelo, R., 2017. Panax ginseng and Panax quinquefolius: from pharmacology to toxicology. Food Chem. Toxicol. 107 (Pt A), 362-372.

Niu, Z.Z., Guo, Y.Y., Zhao, X.L., Jin, H., Gong, Y.L., 2019. Anti-fatigue effect of glutathione in mice and its underlying mechanisms. Food Sci 40 (17), 216-222.

Paschalis, V., Theodorou, A.A., Margaritelis, N.V., Kyparos, A., Nikolaidis, M.G., 2018. Nacetylcysteine supplementation increases exercise performance and reduces oxidative stress only in individuals with low levels of glutathione. Free Radical Biol. Med. 115, 288-297.

Sun, M., Van Wijk, E., Koval, S., van Wijk, R., He, M., Wang, M., Hankemeier, T., van der Greef, J., 2017. Measuring ultra-weak photon emission as a non-invasive diagnostic tool for detecting early-stage type 2 diabetes: a step toward personalized medicine. J. Photochem. Photobiol. B Biol. 166 (complete), 86-93.

Shen, H., Hu, M., Wei, Z.H., Zhang, D., Zhang, J.C., Tian, G.J., 2019. Bile formation, secretion, and excretion and the pathogenesis of cholestasis. J. Chin. Hepatol. 35 (2), 431-437.

Taylor, S.A., Green, R.M., 2018. Bile acids, microbiota and metabolism. Hepatology 68, 1229-1231.

Wu, Y.L., Wang, Q., Chen, Y., Zhang, Z.L., Sun, R.R., Zhao, S.P., Bai, T.T., Li, L.R., 2017. Analysis of qi deficiency constitution related classification and prescription application. Jilin. J. Tradit. Chin. Med. 37 (4), 332-335.

Wang, X.H., Wang, Z.H., Cheng, T., Meng, X.Y., 2018. The relationship between the Qixu syndrome in TCM and disease. Clin. J. Chin. Med. 10 (23), 24-25.

Xing, Y.R., Chen, P., Zhang, R.B., 2015. Recent research progress of interleukin-4. Med. Recapitulate 21 (19), 3457-3461.

Xiong, H., 2012. Differential diagnosis of childhood HyperCKemia. Chin. J. Appl. Clin. Pediatr. 27 (8), 557-559.

Xu, Y., Hou, G.L., Zhou, L.F., Zhang, Y.J., 2018. Application of metabonomics in clinical 
medical research. China. Pharm. 21 (9), 1659-1661.

Yang, S.H., Yin, H., Liu, Y.S., Zhang, J., 2010. Advance in research of interferon gamma. Biotechnol. Bull. 8, 29-34.

Zhao, L., Gao, Y.G., Ji, Q., 2015. Immune effects and mechanism of ginseng. Centr. South Pharm. 13 (7), 741-754.
Zhen, B.X., Jia, L.Q., Yang, G.L., 2017. Study of profile characteristics of serum metabolite in spleen-qi deficient rats treated with Sijunzi Decoction. Chin. J. Tradit. Chin. Med. Pharm. 32 (3), 1234-1237.

Zhu, X.M., 2011. Physiological activity of superoxide dismutase. Contemp. Med. 17 (15), $26-27$. 